\title{
The Effects of Early Pain Experience in Neonates on Pain Responses in Infancy and Childhood
}

\author{
Anna Taddio ${ }^{1,2}$ and Joel $\mathrm{Katz}^{3,4,5}$
}

1 Department of Pharmacy and Research Institute, The Hospital for Sick Children, Toronto, Ontario, Canada

2 Department of Pharmaceutical Sciences, University of Toronto, Toronto, Ontario, Canada

3 Department of Psychology and School of Kinesiology and Health Science, York University, Toronto, Ontario, Canada

4 Department of Anesthesia and Pain Management, Toronto General Hospital and Mount Sinai Hospital, Toronto, Ontario, Canada

5 Department of Anesthesia, University of Toronto, Toronto, Ontario, Canada

\section{Contents}

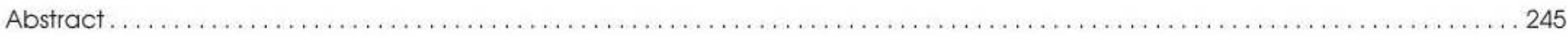

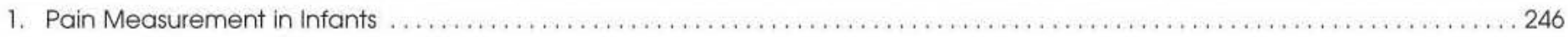

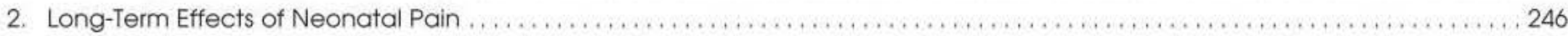

2.1 Birth Experience and Subsequent Pain Responses in Full-Term Infants . . . . . . . . . . . . . . . . . . . . . . . . . 246

2.2 Neonatal Surgery and Subsequent Pain Behaviors in Full-Term Infants . . . . . . . . . . . . . . . . . . . . . .

2.3 Surgery in Early Infancy and Subsequent Pain Behaviors in Full-Term Infants . . . . . . . . . . . . . . . . . . . . . . . 249

2.4 Pathologic Conditions in Full-Term and Preterm Neonates and Subsequent Pain Behaviors .........................249

2.5 Prolonged Hospitalization and Repeated Painful Procedures in Full-Term and Preterm Neonates, and Pain Behaviors Later

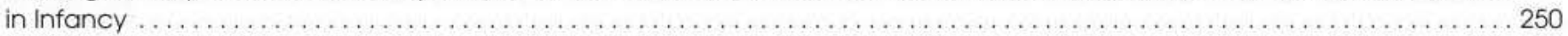

2.6 Pain Responses in Former Preterm Infants After Discharge from Hospital . . . . . . . . . . . . . . . . . . . . . . . . . . . . 252

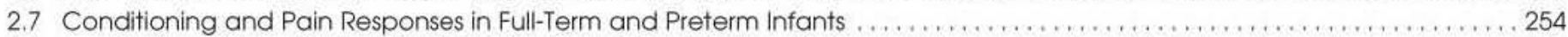

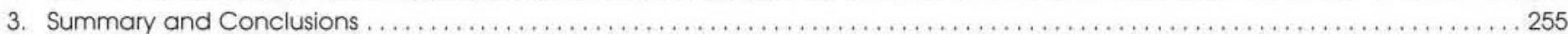

Abstract

Iatrogenic pain is commonplace in newborn infants yet we know very little about its long-term effects. This article reviews the evidence for and against the suggestion that painful procedures experienced in the perinatal period influence subsequent pain responses in infancy or in childhood.

The evidence suggests that early experiences with pain are associated with altered pain responses later in infancy. The direction of the altered response depends, in part, on the infant's developmental stage (full-term vs preterm), and his or her cumulative experience with pain. Preterm infants that are hospitalized as neonates and subjected to painful procedures appear to have a dampened response to painful procedures later in infancy. Full-term neonates exposed to extreme stress during delivery, or to a surgical procedure, react to later noxious procedures with heightened behavioral responsiveness. Studies in which analgesic agents (local anesthetics or opioids) have been administered prior to noxious procedures demonstrate less procedural pain and a reduction in the magnitude of long-term changes in pain behaviors.

The precise determinants of these changes, their extent, and their permanence are not known but they appear to involve noxious stimulus-induced peripheral and central sensitization, as well as classical conditioning. 
The past 2 decades have seen a surge in research examining the long-term effects of pain in the human neonate. The main aim of this review was to determine the impact of a single or repeated painful procedure in the perinatal period on subsequent pain responses in infancy or childhood. The goal was to identify and characterize subsequent pain response patterns in infants and children as a function of prior experience with pain in the neonatal period. The literature examining the long-term effects of neonatal pain experience on other domains of infant development such as childhood behaviors and cognitive abilities has been reviewed in detail elsewhere ${ }^{[1]}$ and is not covered in this article. Cumulative evidence derived from the studies in the present review suggests that untreated pain in the neonatal period may lead to alterations in the pattern of behavioral responses to subsequent painful procedures, although the nature, extent, duration, and clinical importance of these changes remain to be elucidated.

Articles were identified through a database search of MEDLINE from 1966 to 2005 using the following keywords/terms in various combinations: 'analgesia', 'circumcision', 'heel lance', 'hyperalgesia', 'infant', 'long term effects', 'neonate', 'newborn', 'pain', 'pain hypersensitivity', 'pain management', 'postoperative pain', 'procedural pain', and 'surgery'. Relevant articles identified by the database search were obtained and additional references were identified from a review of the bibliography sections.

\section{Pain Measurement in Infants}

This section provides a brief overview of pain measurement in infants. The tremendous advancements over the past 25 years in the knowledge and management of pain are in large part due to our ability to identify and measure pain. Pain measurement is essential to our understanding of the constellation of behaviors that are involved in the responses to noxious procedures. Reliable and valid measurements of pain are important in determining the intensity, quality, and time course of pain; aiding diagnosis; helping to decide on the nature of treatment; and evaluating the effectiveness of selected interventions. ${ }^{[2]}$ Pain measurement is all the more important in infants since unlike adults, infants are incapable of linguistic expression to convey the many qualities and varieties of the pain experience. ${ }^{[3]}$ Therefore, the study of pain in infants will not have a scientific foundation without the use of reliable, valid, and comprehensive measurements of pain.

Research on neonates and infants during acutely painful procedures has identified consistent and reliable responses across three domains: behavioral, physiologic, and neurochemical. ${ }^{[4]}$ The responses to acute pain in neonates and infants are similar to those observed in children and adults. They are generally short-lived and designed to be protective. Behavioral responses include facial grimacing, crying, and body writhing movements. Physiologic responses include changes in heart rate, oxygen saturation, blood pressure, and respiratory rate. Neurochemical responses include the release of a large number of hormones including cortisol, insulin, glucagon, endorphins, and catecholamines. In general, infant responses across the different domains are inter-correlated. ${ }^{[5]}$ As such, a number of composite measures have been developed that incorporate responses from one or more domains to generate an overall pain score. Examples of neonatal and infant pain assessment tools include the Neonatal Facial Coding System, ${ }^{[6]}$ and the Premature Infant Pain Profile. ${ }^{[7]}$

There are fewer data on infant responses to more persistent or long-lasting pain. Persistent pain can occur in certain conditions that involve inflammation, ${ }^{[4]}$ such as osteomyelitis and necrotizing enterocolitis. In addition, persistent pain may be iatrogenic and result from repeated medical procedures (e.g. heel lancing). In adults, chronic pain has been demonstrated to be accompanied by altered sensory states such as hyperalgesia and allodynia. Similar states are likely to develop in newborn infants. In fact, newborn infants may be particularly sensitive to the effects of pain because of rapid brain growth and the potential to permanently alter neuronal development and organization.

\section{Long-Term Effects of Neonatal Pain}

In this section, we review the avenues of research that deal with the effects of pain in neonates on subsequent pain responses in infancy, including the effects of the birth experience, neonatal surgery, pathologic conditions, prolonged hospitalization, and repeated painful procedures, as well as the effects of analgesic drugs. We also evaluate the literature documenting the nature of pain responses in former preterm infants after discharge from hospital. Finally, the role of neonatal classical conditioning on pain responses in full-term and preterm infants is reviewed.

\subsection{Birth Experience and Subsequent Pain Responses in Full-Term Infants}

The possibility that perinatal noxious or traumatic events influence subsequent pain behaviors later in infancy has been investigated in neonates who sustained brachial plexus injury during delivery. ${ }^{[8]}$ Brachial plexus injury, as a result of damage to the cervical nerves due to shoulder dystocia, occurs in $0.1-4 \%$ of births; the risk factors appear to be breech delivery and birthweight $>4 \mathrm{~kg} .{ }^{[9]}$ Lesions vary in severity and may involve only the nerves 
that are distal to the dorsal root ganglion (i.e. post-ganglionic peripheral rupture) or those proximal to the dorsal root ganglion (i.e. pre-ganglionic central axotomy). The extent of neurologic injury determines the clinical presentation as well as the long-term prognosis. ${ }^{[9]}$

Anand and $\mathrm{Birch}^{[8]}$ investigated whether neonatal unilateral brachial plexus injury at delivery led to long-term chronic pain syndromes. Validated tests of sensory and sympathetic function were used to assess 24 patients 3-23 years after brachial plexus injury at birth. These tests included heat, touch and vibration sensation, sweating, and motor function. Both affected and contralateral, clinically unaffected sites were studied. Surgical intervention (i.e. nerve re-innervation) corrected the injury in the majority of patients and was accompanied hy restored sensory function. There was no evidence of chronic pain behavior or neuropathic pain syndromes, and pain responses were normal when noxious test stimuli were applied in unaffected regions. Responses were compared with values for healthy children and adults. These results are in marked contrast to those obtained from adults who sustained similar injuries in adulthood, with or without surgical repair. ${ }^{[8]}$ Adults often misidentify the stimulus modality, show deficits in stimulus localization, and demonstrate allodynia and/or hyperalgesia after re-innervation. The authors speculated that relative neuronal immaturity in the newborn and CNS plasticity are protective against subsequent development of chronic pain syndromes. ${ }^{[8]}$

In contrast, McCann et al. ${ }^{[10]}$ reported the results of a larger, retrospective study of 280 patients, which suggested a cumulative incidence of pain of $3.9 \%$ an average of 17 months after perinatal brachial plexus injury. In $>50 \%$ of the patients, pain was inferred from self-report, parental report, or the surgeon's report. In addition, all patients exhibited a form of self-mutilation behavior, termed autotomy, ${ }^{[1]]}$ frequently observed to be a response to pain or dysesthesias in animals who have undergone experimental deafferentation due to peripheral nerve injury or nerve section ${ }^{[12]}$ and occasionally exhibited in human adults ${ }^{[13\rceil}$ and children ${ }^{[14]}$ in pain. The self-mutilation behavior recorded among the children studied by McCann et al. ${ }^{[10]}$ included excessive mouthing, biting and/or loss of portions of the digits due to excessive biting, prompting evaluation and treatment by a multidisciplinary pain service. The self-injurious behavior was associated with surgical interventions including bone and/or tendon surgery and microsurgical repair of the brachial plexus. For example, the incidence of self-mutilation behavior among children who had had surgery was $6.8 \%$, significantly greater than the incidence (1.8\%) among children who had not had surgery. However, more striking, was the $29.1 \%$ incidence of self-injurious behavior among chil dren who had undergone microsurgical dissection and lysis of adhesions surrounding the brachial plexus with or without nerve grafting. On average, the self-mutilative behavior began 8 months after surgery and did not exceed 6 months in duration. The authors speculated that since only children with the most severe perinatal brachial plexus injuries undergo microsurgical dissection, the greater incidence of self-mutilation may be a result of the prior injury, the surgical procedure, or both.

Al-Qattan ${ }^{[15]}$ reported autotomy in $4.9 \%$ of 127 consecutive cases of perinatal brachial plexus injury. The children ranged in age from 2 to 8 years at the time of report. Self-mutilation behavior was more severe and more prevalent among children with total palsy $(10.8 \%)$ than those with upper or Erb palsy (2.2\%). In contrast with the study by McCann et al., ${ }^{[10]}$ none of the patients studied by Al-Qattan ${ }^{[15]}$ reported pain; there was no evidence that the author inquired about the presence of pain.

In summary, perinatal brachial plexus injury results in selfmutilation behavior months to years after birth in $3.9-4.9 \%$ of patients. Although not all children exhibiting self-mutilation behavior report pain, pain appears to be the trigger for self-mutilation in some cases. The incidence, age at onset, and duration of selfmutilation suggest that the absence of reports of pain by Anand and Birch $^{[8]}$ is likely due to insufficient power to detect the behavior given the sample size of only 24 patients and the 3 - to 23-year assessment period since the injury occurred.

In addition to research on brachial plexus injury, the mode of delivery has been investigated as a potential mediator of subsequent pain responses. Grunau et al. ${ }^{[15]}$ examined the relationship between perinatal variables (mode of delivery, obstetric medication) and neonatal pain expression during a heel lance performed on the second day of life in 140 neonates. Greater use of obstetric medication (opioids, and epidural or general anesthesia) and more stressful deliveries (vaginal delivery with forceps, unplanned caesarean section) were associated with increased neonatal facial activity during heel lance. As the authors note, the type of delivery and use of medication are confounded by the outcome, since more difficult deliveries are associated with increased medication use Nevertheless, either increased use of obstetric medication, more stressful deliveries, or some combination of these two variables appears to be predictive of a heightened pain response up to 2 days later.

Several investigators have examined the hypothalamic-pituitary-adrenal (HPA) system reactivity to pain and stress in infants. Cortisol is the primary glucocorticoid of the HPA system and has thus been used as a marker of HPA axis reactivity. ${ }^{[17]}$ Cortisol 
levels have been measured in the plasma or saliva immediately before and 20-30 minutes after the onset of a painful or stressful event (i.e. at the point in time when they are expected to reach peak levels). Gunnar et al. ${ }^{[18]}$ examined cortisol responses according to birth characteristics (as assessed by the Obstetric Complication Scales $\left.{ }^{[19]}\right)$ in 141 full-term neonates after either two mock discharge examinations (a handling stressor) or two heel lances (a nociceptive stressor) separated by a 24 -hour period. The sample was divided into infants with optimal birth characteristics and those with suboptimal birth characteristics. Sample characteristics of the newborn infants with suboptimal obstetric scores included: birthweight $<2900 \mathrm{~g}$ or $>4000 \mathrm{~g}$, gestational age $<38$ weeks or $>42$ weeks, emergency caesarean section, 5-minute Apgar score $<8$, and neonatal complications after delivery.

In the sub-sample of healthy infants with optimal birth characteristics, there was a significant elevation in cortisol levels in response to the handling stressor after the first discharge examination, but not the second, suggesting an habituation response. ${ }^{[18]}$ This pattern of response was in marked contrast with the heel lance, which showed a significant increase in cortisol release on successive days, with a trend for a greater increase on the second day compared with the first day. The increased response to the second heel lance suggests a sensitization of the HPA axis response. The pattern of response to the discharge examination differed for infants with suboptimal birth characteristics. In this sub-sample, habituation did not occur in response to the second discharge examination. However, the greater response to repeated heel lance was comparable to that observed in neonates with optimal birth characteristics. These data suggest that HPA axis responses are stressor specific and that regulatory processes may be disrupted in infants with suboptimal birth characteristics.

Two other studies investigated infant cortisol responses during immunization as a function of birth conditions. ${ }^{[20,21]}$ In the first study, suboptimal birth characteristics were related to cortisol responses in 64 infants undergoing routine 2-, 4-, and 6-month vaccination. ${ }^{[20]}$ Infants in the optimal birth characteristics group had a higher cortisol response to immunization at 2 months, whereas in the non-optimal birth characteristics group (defined as head circumference $<25$ th percentile and 1-minute Apgar score $<9$ ), the peak cortisol response occurred at 4 months and 6 months. ${ }^{[20]}$

In the second study, salivary cortisol responses and cry duration in infants undergoing 2-month immunization varied according to mode of delivery. ${ }^{[21]}$ Neonates were separated into three delivery groups: normal vaginal delivery $(\mathrm{n}=46)$, assisted delivery (ventouse or forceps, $n=20)$, and elective caesarean section $(n=$
10). Changes in cortisol levels were greatest in the assisted delivery group and lowest in neonates born by caesarean section. The duration of infant crying showed a similar pattern. ${ }^{[21]}$ These results suggest that the mode of delivery (and associated stress) may influence later pain responses.

In summary, birth characteristics appear to influence neonate and infant pain response patterns. Highly stressful deliveries or suboptimal birth characteristics are associated with exaggerated subsequent responses to painful procedures, which are observed up to the age of 6 months.

\subsection{Neonatal Surgery and Subsequent Pain Behaviors in Full-Term Infants}

Two studies have investigated the effect of neonatal surgery on infant pain responses during subsequent routine immunization. ${ }^{[22-24]}$ The first study involved a post-hoc statistical analysis of a randomized controlled trial by Taddio et al. ${ }^{[22]}$ Male infants who had been circumcised without anesthesia as neonates showed a heightened behavioral pain response to subsequent 4- or 6-month immunization pain compared with male infants who had not undergone neonatal circumcision. These results suggested that neonatal male circumcision may be associated with alterations in pain behaviors later in infancy but this conclusion was tentative because the analysis was retrospective and the sample size was small $(\mathrm{n}=42)$.

The investigators subsequently performed a prospective study examining whether neonatal circumcision altered the pain response at 4- or 6-month immunization and whether pretreatment of circumcision pain with a topical anesthetic (lidocaine/prilocaine cream) could prevent subsequent changes in the pain response at immunization ${ }^{[23]}$ In total, 87 infants participated in the study. The infants formed three groups: uncircumcised, circumcised with lidocaine/prilocaine, and circumcised with placebo. Infants were observed during routine immunization and their pain was assessed from videotaped records using three behavioral measures: facial grimacing, percentage of time crying, and visual analog scale scores rated by trained, blinded observers. For all three measures, circumcised infants demonstrated a significantly stronger pain response to routine vaccination compared with uncircumcised infants. Visual analog scale scores were $65-117 \%$ higher for the infants circumcised with placebo (5.1) compared with the uncircumcised infants (3.1), and circumcised infants given preoperative lidocaine/prilocaine (3.3) had significantly lower visual analog scores than circumcised infants given placebo preoperatively. These results suggested that circumcision in male infants was 
associated with increased pain responses to immunization 4-6 months after surgery and that local anesthesia administered before circumcision partially attenuated the development of these behaviors. The authors raised the possibility that the observed effects may have been due to baseline differences between infants who were circumcised and those who were not, or differences in family dynamics post-circumcision, but were unable to find significant intergroup differences in demographic characteristics or infant temperament that would explain the results. ${ }^{[23]}$

\subsection{Surgery in Early Infancy and Subsequent Pain Behaviors in Full-Term Infants}

Peters et al. ${ }^{[24]}$ compared pain responses in two groups of 50 infants at either the 14-month measles-mumps-rubella immunization or the 45-month diphtheria-tetanus-trivalent polio immunization. One group had undergone major surgery in the first 3 months of life; the other group consisted of control infants with no history of surgery. The group that had undergone major surgery (index group) was comprised of infants who had participated in a previous clinical trial involving morphine administration for postoperative analgesia after major abdominal or thoracic surgery. In that trial, infants had received morphine for postoperative pain control either by continuous infusion or by intermittent administration (every 3 hours); no differences in efficacy were observed between groups. ${ }^{[25]}$ The control infants in the study by Peters et al. ${ }^{[24]}$ were matched to the index group by type of immunization and community healthcare pediatrician. There were no differences between groups in facial pain responses, heart rate, or salivary cortisol levels during the first or second immunization. The authors concluded that morphine administration in the index group had prevented the development of alterations in pain threshold in the long term. However, within the index group, investigators found that the number of adverse hospital experiences (major surgery, Therapeutic Intervention Scoring System ${ }^{[26]}$ score, and number of days in the neonatal intensive care unit [NICU]) correlated positively with facial pain responses and negatively with heart rate responses during immunization at 14 months of age but not at 45 months of age. Cortisol levels did not correlate significantly with adverse hospital experiences at either point in time. The authors concluded that the apparent alterations in pain response due to neonatal surgery that were observed at 14 months of age were not permanent since they were not observed at 45 months of age. The authors did not address the questions of why infants of such vastly different ages and those receiving different vaccines were grouped together in the same analysis since both age (14 vs 45 months) and type of vaccine administered influence infant pain responses Separate analyses comparing index and control infants during the 14- and 45-month immunizations were not conducted.

In another study by the same authors, ${ }^{[27]}$ the effect of prior surgery on perioperative pain responses during and following a subsequent surgical procedure was evaluated. One hundred and sixty-four infants born at $\geq 36$ weeks' gestation were assigned to one of three groups according to their past surgical history. Infants in group $1(n=129)$ were scheduled for surgery for the first time and served as the control group. Infants in groups $2(n=13)$ and 3 $(\mathrm{n}=22)$ had undergone one previous surgery either in the same (group 3) or a different (group 2) dermatome from the second upcoming surgery. The median number of weeks since the first surgery was 10 (range: $3-34$ ) and 15 (range $8-70$ ) for groups 2 and 3 , respectively. The median age of the infants in weeks was 4 (range 0-63), 21 (range 4-104), and 17 (range 8-70) for groups $1-3$, respectively. The following results were obtained after statistically adjusting for gestational age, postnatal age, and severity of surgical stress. Intraoperative fentanyl requirements, and plasma epinephrine and norepinephrine concentrations were significantly higher in group 3 compared with groups 1 and 2; there were no significant differences between groups 1 and 2. Postoperative morphine requirements were significantly higher in groups 2 and 3 compared with group 1 . In addition, postoperative pain, as measured by validated (i.e. visual analog and COMFORT ${ }^{[28]}$ ) scales, was significantly higher in group 3 compared with group 1 (but not group 1 vs group 2). The authors concluded that surgery in early infancy is associated with prolonged pain hypersensitivity in response to a subsequent surgical procedure performed in the same dermatome but the magnitude of the effects was not deemed to be clinically relevant.

\subsection{Pathologic Conditions in Full-Term and Preterm Neonates and Subsequent Pain Behaviors}

$\Lambda$ recent three group study investigated long term changes in cutaneous abdominal sensitivity in infants with pathologic abdominal disease and presumed abdominal visceral pain. ${ }^{[29]}$ Group 1 comprised newly born preterm and full-term neonates 30-41 weeks' post-conceptional age $(n=25)$; group 2 comprised infants with unilateral hydronephrosis (UH) 2-95 weeks' post-conceptional age $(n=30)$; and group 3 comprised healthy control infants 42-95 weeks' post-conceptional age $(n=52)$. Using nylon von Frey hairs (monofilaments of varying diameters designed to apply fixed forces to the skin), graded mechanical pressure was applied to the surface of the abdomen on either side of the midline, lateral 
to and at the level of the umbilicus. The von Frey hairs were applied in ascending order until contraction of the ipsilateral abdominal musculature was observed. The force associated with the von Frey filament that elicited the contraction was defined as the threshold for the abdominal skin reflex (ASR). Neonatal and control infant responses were examined on a single occasion; the infants with UH were examined at their first appointment in the urology clinic and again 3 months postoperatively, if surgery had been performed. The antero-posterior diameter of the renal pelvis was used as a marker of the degree of hydronephrosis for infants with UH. The results of the urologic investigations as well as the side affected were not disclosed to study observers during the trial.

ASR thresholds increased and reflex hip flexion responses decreased significantly as a function of post-conceptional age for neonates and control infants. ${ }^{[29]}$ In addition, ASR thresholds were similar on both sides of the abdomen in most neonates and control infants. Infants with UH showed no changes in abdominal sensitivity or hip flexion responses with increasing post-conceptional age, even when tested on the unaffected side. The majority of infants with UH had lower ASR thresholds on the side of the abdomen with the hydronephrotic kidney and the magnitude of the reflex radiation (i.e. hip flexion) was also greater on the side of the abdomen with the hydonephrotic kidney compared with the unaffected side. The degree of pelvic dilatation was not found to influence the ASR threshold in infants with UH. Four of five infants with UH that had undergone corrective surgery and were seen at their 3-month postoperative visit showed lower ASR thresholds (on affected and unaffected sides) compared with control infants of the same age range.

In summary, the ASR threshold and the reflex radiation decreased in neonate and control infants as they matured from 30 to 95 weeks' post-conceptional age, reflecting a decrease in abdominal sensitivity. Infants with UH displayed lower ASR thresholds and greater reflex radiation compared with unaffected infants, suggesting that the usual developmental progression is altered and that infants with UH have increased abdominal sensitivity. Moreover, although skin sensitivity was greater on the affected side, the ASR thresholds were lower on both sides of the abdomen. The increased abdominal sensitivity appeared to be long-lasting, persisting up to 3 months after corrective surgery and is suggestive of visceral hypersensitivity. ${ }^{[29]}$

\subsection{Prolonged Hospitalization and Repeated Painful}

Procedures in Full-Term and Preterm Neonates, and Pain Behaviors Later in Infancy

Iatrogenic pain is commonplace in hospitalized infants. It has been hypothesized that repeated pain experienced during the perinatal period may have long-lasting effects especially in preterm infants, due to underlying immaturity in pain modulating systems. ${ }^{[30]}$ Several groups of investigators have examined pain responses in infants who, as preterm or full-term neonates, had undergone multiple painful procedures during hospitalization in the NICU. ${ }^{[31-35]}$ The results show that both behavioral and physiologic responses to pain may be altered over time although the direction of the changes that are observed is not always consistent for a given variable. In addition, changes in pain response were not consistently predicted by the number of painful procedures to which the infants were exposed. Differences in study designs and environmental conditions may explain the inconsistent findings. These studies are described in more detail below.

The relationship between infant characteristics and NICU experiences on pain responses during heel lance has been investigated in two studies. ${ }^{[31,32]}$ In the first study, ${ }^{[3]]}$ behavioral and physiologic pain responses of preterm infants born at 32 weeks' gestational age were compared with those of preterm infants aged 32 weeks who had been born 4 weeks earlier. Eighty-nine infants were observed during a routine heel lance. The earlier born infants demonstrated significantly less behavioral grimacing (i.e. brow bulge, eye squeeze, naso-labial furrow) compared with the newly born infants. Lower Apgar scores at birth and a greater number of invasive procedures significantly predicted heightened behavioral pain responses. Earlier born infants exhibited significantly higher maximum heart rates and lower oxygen saturation values than the newly born infants; physiologic responses correlated significantly with birthweight and gestational age at birth. The authors concluded that preterm infants who spent 4 weeks in the NICU differed in their pain responses when compared with newly born infants of the same post-conceptual age. Earlier born infants had a profile of responsiveness to pain that seemed immature compared with the later born infants.

In another study, Grunau et al. ${ }^{[32]}$ investigated the behavioral and physiologic responses of 136 low birthweight infants $(\leq 1500 \mathrm{~g})$ at 32 weeks' post-conceptional age during a heel-lance procedure. Infant responses (facial activity and heart rate) increased during the procedure suggesting they experienced pain. Regression analyses demonstrated that the number of invasive procedures was negatively associated with behavioral and heart 
rate variability. In addition, lower gestational age at birth and prior dexamethasone exposure were associated with reduced facial responses, and lower gestational age at birth and the number of days of dexamethasone were associated with reduced heart rate variability (as assessed by spectral analysis). However, increased heart rate variability was associated with increased exposure to morphine. The authors suggested that increased exposure to painful procedures diminished the pain response and that morphine exposure partially prevented the diminished heart rate variability component of the pain response. Infants undergoing $\leq 20$ painful procedures differed in their responses compared with those undergoing $>20$. The finding that exposure to a greater number of painful procedures is correlated with diminished facial reactivity is consistent with the previous study by Johnston and Stevens. ${ }^{[31]}$ However, Johnston and Steven ${ }^{[31]}$ observed a positive relationship between maximum heart rate and greater early pain exposure whereas Grunau et al. ${ }^{[32]}$ found a negative relationship between heart rate variability and number of painful procedures. The difference in the direction of the relationship between pain responsivity and cardiac measures may reflect underlying differences in the infant samples, cardiac measures, or study designs.

Most recently, Grunau et al. ${ }^{[36]}$ evaluated the effects of repeated neonatal pain and morphine exposure on subsequent plasma cortisol responses to handling and pain responses (behavioral and cardiac reactivity) to noxious cutaneous procedures in 87 preterm infants from two age groups: extremely low gestational age (23-28 weeks' gestation) and very low gestational age (29-32 weeks' gestation). All neonates were studied at 32 weeks' gestational age. In the extremely low gestational age group, a significant negative correlation was observed between the number of skin-breaking procedures and (i) the plasma cortisol response to handling $(\mathrm{r}=-0.5)$ and (ii) the lower facial reactivity to noxious cutaneous procedures $(r=-0.44)$. Morphine exposure was also significantly negatively associated with lower facial activity $(\mathrm{r}=$ -0.42 ), but not plasma cortisol response in the younger gestational age group. In contrast, correlational analyses did not reveal any significant relationships among these variables (number of skinbreaking procedures, morphine exposure, cortisol response, pain response) in the very low gestational age group. There were no significant correlations between the number of skin-breaking procedures and morphine exposure or cardiac reactivity during painful procedures in either age group. In addition, cumulative procedural pain was associated with a diminished behavioral response to pain. The authors concluded that a greater number of skinbreaking procedures in physiologically immature neonates results in an early dampening of the HPA axis or the duration of NICU hospitalization, followed by an upregulation of the HPA axis by 8 months of age. ${ }^{[37]}$ The finding that morphine did not appear to ameliorate the effects of repeated pain on behavioral reactivity as it did in an earlier study by the same group ${ }^{[32]}$ was explained by the absence of dexamethasone exposure since birth in neonates in the more recent study. ${ }^{[36]}$

Three longitudinal studies have demonstrated that pain responses change over time in preterm infants exposed to repeated painful procedures as neonates. ${ }^{[33-35]}$ Fitzgerald et al. ${ }^{[33]}$ used the flexion reflex threshold (a nociceptive reflex involving noxious stimulus-elicited limb withdrawal) and found that tactile responses were heightened in preterm infants who had undergone repeated painful procedures. The application of graded von Frey hairs to the lateral plantar surface of the foot near the heel was used to elicit the flexor reflex.

A total of 17 preterm infants born at 27-32 weeks' gestation participated in a double-blind study. ${ }^{[33]}$ Flexor reflex thresholds were obtained regularly over a period of 1-4 weeks' postnatally. During this period, routine heel lances (performed approximately every 4 hours for clinical purposes) were performed on one foot only. The mean withdrawal thresholds were then compared among three treatment groups of infants that received: (i) heel lances only; (ii) heel lances with a placebo anesthetic cream; or (iii) heel lances with a local anesthetic cream (lidocaine/prilocaine). The mean threshold of the flexor reflex evoked from heel stimulation was significantly lower for the heel-lanced foot of infants who received placebo or nothing at all compared with the intact contralateral side. In infants who received local anesthetic, the flexor reflex threshold on the injured foot was significantly higher than that in infants who received placebo or nothing, and not significantly different from the non-injured side.

The results of the study by Fitzgerald et al. ${ }^{[33]}$ suggested that the lower threshold in the area of tissue damage was accompanied by hyperalgesia; that is, increased pain to a normally painful stimulus. This hypersensitivity was prevented by pretreatment of the damaged area with the topical anesthetic cream. While not a direct measure of pain, withdrawal responses in infants vary positively with the presence of tissue injury and are likely to be correlated with pain, as they are in adults.

Johnston et al., ${ }^{[34]}$ prospectively examined changes in pain response over time in a cohort of 28 preterm infants of $<30$ weeks' gestational age at birth. Pain responses were observed during sham and real heel-lance procedures conducted at approximately 2-week intervals over an 8-week period. Infant facial grimacing and maximum heart rate change were significantly greater during the real procedure compared with the sham procedure across all 
timepoints. Behavioral responses increased over time, possibly due to maturation of the nervous system although heart rate remained relatively constant. Oxygen saturation values did not change as a function of type of procedure or time. The number of invasive procedures was not associated with any of the outcomes as it was in the previously reviewed study by these investigators. ${ }^{[31]}$

Porter et al. ${ }^{[35]}$ also used a longitudinal design to evaluate pain responses over time in preterm infants born at $<28$ weeks' gestation. The infants were followed as they progressed through four conceptional ages: $<28$ weeks, $28-32$ weeks, $32-36$ weeks, and $>36$ weeks. Unlike previous studies, procedures of varying degrees of invasiveness were included, from mildly invasive (e.g. gavage tube insertion), to moderately invasive (e.g. venous puncture) and highly invasive (e.g. lumbar puncture). Mean heart rate change (from baseline phase to procedure phase) increased with increasing conceptional age. In addition, procedural invasiveness significantly predicted the magnitude of infant behavioral and physiologic responses for mildly invasive procedures compared with moderately and highly invasive procedures, whereas responses to moderately and highly invasive procedures did not differ. Thus, as the time in hospital increased, infant responses became increasingly more vigorous. However, in the absence of a control group, it is not clear whether the increases in pain responses over time stem from the history of prior painful procedures, NICU exposure, maturation, or some combination of these variables.

In summary, preterm and full-term infants that have spent time in the NICU and undergone repeated painful procedures appear to show changes in pain responses over time. However, the direction of altered responsivity is not consistent across studies and is possibly influenced by differences in the timing of painful injuries. ${ }^{[38]}$ In general, behavioral reactivity appeared to be lower in younger gestational age infants and higher in older gestational age infants. The study by Fitzgerald et al. ${ }^{[33]}$ is the only one to have controlled for the extent of noxious stimulation across groups of infants. In the other uncontrolled studies, ${ }^{[31,32,34,35]}$ the observed changes in pain responsivity over time cannot be unambiguously attributed to the prior painful experiences given the absence of appropriate control conditions.

\subsection{Pain Responses in Former Preterm Infants After Discharge from Hospital}

Pain behaviors have been evaluated after discharge from the NICU in a single cohort of former extremely low birthweight
(ELBW) infants $(\leq 800 \mathrm{~g})$ undergoing a finger lance on two occasions 4 months apart. ${ }^{[39,40]}$ All infants were participating in a study of iron metabolism and underwent a finger lance for blood collection.

In the first study, finger lance pain responses (facial grimacing, heart rate variability) in 24 former ELBW infants were compared with those of 21 term-born control infants at 4 months (corrected age). ${ }^{[39]}$ Spectral analysis was used to evaluate the contribution of the sympathetic and parasympathetic components of heart rate variability. Overall, there were no differences in facial pain scores or mean heart rate between the groups. However, subtle differences in facial responses and cardiac autonomic responses were observed. For facial activity, there was a trend toward less facial activity in the recovery phase of the procedure for ELBW infants, suggesting a more rapid return to baseline after the lance was completed. For cardiac autonomic responses, there were differences in sympathetic and parasympathetic activation, including a less intense parasympathetic withdrawal in the lance phase in ELBW infants and a sustained sympathetic response during the recovery phase. For the ELBW group, infants that demonstrated earlier termination of facial grimacing responses during the recovery phase also had less intense sympathetic and parasympathetic responses to the painful event than infants that exhibited late facial grimacing recovery. Early recovery was also associated with longer stays in the NICU, increased severity of illness, a greater number of procedures, and significantly more intravenous morphine.

In summary, physiologic and behavioral responses were similar between former ELBW infants and term-born control infants at 4 months (corrected age) ${ }^{\left[{ }^{[3]}\right.}$ However, subtle differences in cardiac autonomic and facial responses were observed. The following factors were discussed to account for the few lasting differences in pain response.

- Liberal use of analgesia in the study cohort, thereby preventing any long-term effects of pain in the ELBW infants.

- Ability of the pain system to overcome the effects of early repeated noxious events.

- Examination of a site (finger) remote from the area of early injury (i.e. heel) induced during the NICU stay.

The authors also raised the issue of what the most appropriate control infant is for an ELBW infant. It is not known if term infants of the same corrected age (4 months) are appropriate controls for former ELBW infants, who were approximately 7 months ex-utero at the time of this study. 
In a follow-up study, ${ }^{[40]}$ ELBW infants were compared with control term-born infants at 8 months (corrected age). All infants were taking part in the same study of iron metabolism that required a second finger lance at 8 months (corrected age). There were no differences in facial grimacing or cardiac responsivity between the groups. However, as in the previous study, ${ }^{[39]}$ ELBW infants exhibited subtle differences in responses compared with control infants, including higher basal heart rates and qualitative differences in their patterns of behavioral and physiologic recovery. Compared with control infants, ELBW infants had a quicker facial grimacing response during finger lancing and a faster reduction in facial activity, suggesting that nociceptive processing speed was increased. They also showed reduced sympathetic and greater parasympathetic modulation. In the ELBW infant group, the number of previous invasive procedures since birth was significantly correlated with reduced facial grimacing and reduced heart rate change during the finger lance and the recovery phases. Heart rate change during the recovery phase was related to the number of days in the NICU, number of surgical procedures, and opioid exposure. Greater overall exposure to morphine was associated with a pattern of heart rate recovery that resembled that of termborn infants.

The authors concluded from both studies ${ }^{[39,40]}$ that although overall reactivity did not differ significantly between ELBW infants and term-born control infants, there were subtle differences that appeared to increase over time. At 4 months, there was a trend toward faster recovery for ELBW infants than control infants and, by 8 months, ELBW infants recovered faster, both behaviorally and physiologically. ELBW infants showed a lower basal resting heart rate, less cardiac autonomic reactivity to heel lance, and faster autonomic and behavioral recovery than term-born control infants. It appears that cumulative painful procedures in former preterm infants are associated with decreased responses to pain later on in infancy.

More recently, Buskila et al. ${ }^{[4]]}$ evaluated the pain responses to pressure point stimulation using a dolorimeter in 60 adolescents who had been very low birthweight (VLBW) infants and in 60 control adolescents who had been born at full term. Hospital records showed that at birth, the VLBW infants weighed significantly less than the full-term control infants (1232g vs $3491 \mathrm{~g}$ ) and had spent an average of 65 days in the NICU. As adolescents, the formerly VLBW infants showed significantly more tender points as well as significantly greater tenderness (lowered threshold) in response to pressure applied using the dolorimeter than the formerly full-term control infants. The two groups did not differ significantly on other measures of pain prevalence or severity, which were near zero in both groups. The authors raised the possibility that the altered pain sensitivity to pressure observed in the VLBW group may put them at greater risk for later development of pain problems. Although the authors appear to attribute the adolescent pain hypersensitivity specifically to the neonatal pain experienced by the formerly VLBW infants while in the NICU, many other factors may be involved including other biologic, social, and environmental influences. In addition, the possibility of measurement bias cannot be ruled out because there is no mention of whether the pain assessments were conducted with the assessor blind to the adolescents' birth status. Sampling bias may also limit interpretation since age-matched control individuals were selected from among the friends of the index group.

It should be noted that pain response patterns of newly born infants vary according to gestational age and that some of the results may be more closely related to having been born prematurely rather than to pain or pain experiences. In a study of infants of gestational age 25-41 weeks and $\leq 1$ week postnatal age, Craig et al. ${ }^{[42]}$ demonstrated that greater gestational age was associated with increased behavioral (facial and body movements) reactivity to heel lance. In fact, response to heel lance of infants in the youngest gestational age group was indistinguishable from the baseline phase of the procedure. Although physiologic measures changed relative to baseline during the procedure, they were not related to gestational age. However, there was a general trend toward an inverse relationship between gestational age and heart rate during heel lance, with younger infants demonstrating greater increases in heart rate compared with older infants. The pattern of physiologic response appeared to be less specific than the behavioral response to the different phases of the procedure, and more closely related to cumulative stress evoked by the entire procedure.

It has been postulated that infant handling just prior to heel lancing is an important predictor of responsivity to subsequent painful procedures. In a study by Porter et al., ${ }^{[43]} 48$ preterm and full term infants in the first 4 days of life were randomly assigned to either 'no intervention' or a 'handling procedure' prior to heel lancing. Infants in the handling group showed significantly more facial grimacing and higher heart rate during the heel lance compared with non-handled infants. However, the number of previous painful procedures did not significantly predict the pain response. Another study by Johnston et al. ${ }^{[44]}$ yielded somewhat different results. The study was a secondary analysis of a clinical trial. A total of 120 preterm infants (average gestational age 28 weeks) were included: 96 had demonstrated a pain response during a heellance procedure and 24 had not. Pain was assessed using the 
Premature Infant Pain Profile. ${ }^{[7]}$ Logistic regression analysis indicated that the factors significantly associated with a decreased pain response were younger age (post-conceptional age at birth and postnatal age), being asleep during the procedure, and having undergone a painful event more recently (i.e. within 5 hours). Interestingly, the number of previous invasive procedures was not associated with the pain response.

The results from the study by Johnston et al. ${ }^{[44]}$ are consistent with earlier findings of a dampened, or less robust, response to painful procedures in younger and therefore, less mature infants, ${ }^{[42]}$ as well as in sleeping infants. ${ }^{[6]}$ However, they are not entirely consistent with respect to (i) the lack of a relationship between the number of previous noxious procedures and the pain response and (ii) a reduced response in the presence of recent painful procedures. The authors speculated that infants having undergone more recent invasive procedures (within 5 hours) may not have been afforded sufficient recovery time to mount a response to the subsequent heel lance, although the mean time difference between the heel lance and the next most recent invasive procedure was 10 hours. In addition, as in the study by Porter et al. ${ }^{[43]}$ infants may not have been exposed to a sufficient number of noxious events for a relationship to have developed between the number of painful procedures and the pain response.

\subsection{Conditioning and Pain Responses in Full-Term and Preterm Infants}

There are anecdotal reports suggesting that preterm and fullterm infants can learn to anticipate an impending noxious event. ${ }^{[45-}$ ${ }^{47]}$ These reports imply that the nature of the learning involves a form of classical conditioning in which a previously neutral, nonnoxious stimulus (e.g. gentle touch), which reliably precedes a noxious procedure (e.g. heel lance), acquires the capacity to elicit a response suggestive of pain after one or more pairings of the two stimuli. The current state of the literature does not permit an evaluation of the extent to which increased responsivity to pain in infants undergoing repeated invasive procedures can be attributed to a classically conditioned response (e.g. involving anticipatory pain behaviors), versus noxious stimulus-induced sensitization of nociceptors and/or central sensitization (hyperexcitability) of sensory structures involved in processing somatosensory input. Previous studies have typically investigated infant reactions to a single painful stimulus, ${ }^{[31,32]}$ thereby precluding evaluation of conditioning effects. The few studies that have examined infant pain response to repeated noxious procedures have limited their outcomes to the infants' responses to the noxious event; ${ }^{[34,35]}$ they have not assessed infant behaviors in response to those associated activities that immediately precede or follow the noxious procedure. Thus, it has not been possible to determine the contribution of classical conditioning to overall pain response. Even fewer studies have specifically investigated anticipatory pain responses in newborn infants undergoing painful procedures.

Owens and Todt ${ }^{[48]}$ examined the pain responses of 20 full-term neonates after they had been randomized to one of two test conditions: (i) sham heel lance followed by a real heel lance (for the newborn screening test); or (ii) real heel lance followed by a sham heel lance. The sham heel lance consisted of cleansing the heel with an alcohol pad with no subsequent heel lance, while the real heel lance involved cleansing of the heel followed by a heel lance. The time lag between procedures was $<1$ hour and infants were 30-54 hours of age at the time of testing. The authors postulated that because rubbing the heel with the alcohol pad immediately preceded heel lancing, it had the potential to become a conditioned stimulus. Thus, behavioral responses to skin cleansing would be expected to vary depending on whether infants had had prior experience with heel lancing. The results did not support the hypothesis; previous exposure to heel lance did not lead to an increase in response during heel cleansing. The negative findings may be due to the limited prior experience with heel lancing (only one prior exposure) combined with the small sample size.

Goubet et al. ${ }^{[49]}$ observed the responses of 14 preterm infants (gestational age 28-32 weeks) undergoing repeated heel lances over a period of 2 weeks. The infants' responses during a nonpainful stimulus (picking up the leg) that immediately preceded a heel lance were evaluated. It was hypothesized that if infants learned to predict the impending heel lance, they should increase their responses during the leg pickup over time. The results partly supported the hypothesis. The average heart rate change between the baseline phase and the leg pickup changed over time; infants responded to the leg pickup with an increased heart rate after 2 weeks compared with the baseline response. This suggested that over time picking up the leg became a conditioned stimulus signaling the impending heel lance. However, unlike heart rate, behavioral measures (facial grimacing and body movements) did not change over time. In fact, infants who had undergone a greater number of heel lances by 2 weeks had a tendency to display fewer facial responses than those who had undergone fewer heel lances. A small sample and lack of a control group precluded more extensive analyses and conclusions.

More recently, Taddio et al. ${ }^{[50]}$ observed conditioning and hyperalgesia in full-term newborns exposed to repeated heel lances. Unlike the previous study, ${ }^{[49]}$ these investigators included a 
group of infants that experienced repeated pain and a control group that did not. The two study groups comprised newborn infants born to mothers with diabetes mellitus $(\mathrm{n}=21)$ or to mothers without diabetes $(n=21)$. Infants of mothers with diabetes had, on average, ten heel lances to monitor blood glucose levels prior to a venipuncture performed on the dorsum of the hand for the newborn screening test (second day of life). Control infants did not undergo heel lancing. Investigators compared behavioral pain responses between the two groups during venipuncture using three measures: facial grimacing, percentage of time spent crying, and visual analog scale scores rated from videotapes by blinded observers.

During the preparatory phase of the venipuncture (i.e. cleansing the skin on the hand), facial grimacing pain scores were higher in infants of mothers with diabetes compared with infants of mothers without diabetes ${ }^{[50]}$ Eighty-six percent of infants of mothers without diabetes had visual analog scale pain ratings of $0 \%$ versus $52 \%$ of infants of mothers with diabetes. During venipuncture, infants of mothers with diabetes had higher scores than infants of mothers without diabetes for facial grimacing, visual analog scale, and percentage of time crying. These responses suggested that infants exposed to repeated heel lances learned to anticipate the pain of an impending venipuncture. Moreover, the intensity of the pain they experienced in response to venipuncture was greater than that of infants not exposed to repeated heel lances. Venipuncture pain scores were significantly higher in the infants of mothers with diabetes when pain response during skin cleansing was entered as a covariate in the analysis, suggesting that there was hyperalgesia in addition to anticipatory pain.

There were several limitations that prevented definite conclusions from this study being made, including the relatively small sample size and the lack of measurement of physiologic responses to compare with behavioral responses. ${ }^{[50]}$ Furthermore, the investigators compared pain responses during the first painful procedure performed after birth (intramuscular injection of vitamin K) to rule out baseline differences in pain responses in the different cohorts of infants of mothers with diabetes and infants of mothers without diabetes. Despite these limitations, this study provides preliminary evidence that infants, as young as $24-36$ hours old, exposed to repeated painful procedures can learn to anticipate pain and exhibit increased behavioral responses to a noxious stimulus.

In summary, there is evidence that neonates can learn to anticipate pain. The mechanism for this appears to involve a classically conditioned response involving a previously non-noxious stimulus acquiring the capacity to elicit pain behaviors after repeated pairing with a subsequent noxious procedure. This phenomenon has been observed in neonates in their first day of life. It remains to be determined whether the heightened response to the conditioned stimulus involves the same underlying mechanisms as those involved in pain in response to a noxious procedure, i.e. do infants actually feel pain in response to a non-noxious conditioned stimulus or are our current measurements of infant pain simply not sensitive enough to discern fear and anxiety-related states from pain? In addition, we do not know the minimum number of trials that are necessary before conditioning effects become established, whether the conditioning process is procedure specific, whether certain non-noxious stimuli are more easily conditioned than others, and whether regular use of analgesics would prevent the establishment of aberrant response patterns to subsequent noxious events.

\section{Summary and Conclusions}

Because iatrogenic pain is commonplace in newborn infants, it is generally accepted that pain in newborns should be minimized or prevented if at all possible. Despite this, the routine use of analgesia to prevent procedural pain in newborns has only recently begun to gain momentum. ${ }^{[51-53]}$ This increase in the utilization of analgesics for neonatal pain management is due to the growing body of literature demonstrating that neonates do feel pain as well as international consensus statements promoting the routine use of analgesics in this population. ${ }^{[54,55]}$ In addition, one of the major justifications for the increased use of analgesics in neonates and infants undergoing painful procedures is the accumulating evidence suggesting that untreated pain and stress may have longlasting effects on subsequent pain responses later in infancy.

The studies reviewed in this article show that early experiences with pain are associated with heightened or dampened pain responses later in infancy, depending, in part, on the infant's level of maturity and cumulative experience with pain. Preterm infants that are hospitalized as neonates and subjected to repeated painful procedures appear to have a dampened response to subsequent painful procedures later in infancy. On the other hand, full-term infants exposed to extreme stress during delivery or to a surgical procedure react to later noxious procedures with heightened behavioral responsiveness. In addition, neonates who sustained brachial plexus injury during delivery and who underwent surgical repair without adverse consequences show signs of chronic pain months after surgery.

The conflicting findings among studies may reflect a combination of factors. Firstly, the observed differences in pain responses may in part be accounted for by differences in neonatal character- 
istics (e.g. gestational age, illness severity), concurrent illness, and exposure to local NICU practices (e.g. ventilation strategies, medications used). ${ }^{[56]}$ In addition, variability in the time frame within which infants were observed may explain certain discrepancies between study outcomes. For example, early neonatal pain experience may influence subsequent pain responses later in infancy ${ }^{[23]}$ but not in childhood. ${ }^{[8]}$ Developmental changes in infant pain response capacity may mean that certain behaviors that reflect pain later in infancy may not have developed at earlier conceptional or postnatal ages.

Other factors that may partly explain discrepancies in study outcomes have to do with methodologic and design issues. Studies have utilized different outcome measures and it is not clear whether any or all of these measures capture the aspects of pain expression. In addition, in many studies, although both physiologic and behavioral responses to pain were recorded, these measures did not always correlate with one another and in some cases were discordant. This may reflect the fact that physiologic and behavioral responses to pain are unique, that an individual's response is different for physiologic and behavioral systems, or that there are differences in the ontogeny of these systems or in the effects of pain on the developing infant. ${ }^{[5]}$ One of the most important methodologic differences among studies is the process by which infants were allocated to a treatment group or a non-intervention group. In most studies, infants were not randomly assigned to groups; thus any observed differences in pain response, or the lack of a pain response, may have been due to factors other than the early pain experience.

The nature and extent of prior injury and pain are important factors to control for but this has rarely been done. Pain reactivity is usually determined at a site of previous injury, but the extent of prior injury has not been quantified. The manner by which hyperalgesia due to repeated injury at one body region influences pain response at an alternate location whether in injured or intact tissue remains to be determined. ${ }^{\left[{ }^{[3]}\right.}$ It is possible that effects are most significant in the same region due to peripheral sensitization of nociceptors. In addition, there may be centrally mediated effects, leading to overall changes in pain perception and response independent of original pain location. Finally, the influences of ongoing experience, including conditioning effects, parental, and other environmental factors may have contributed to differential outcomes across the studies reviewed.

Despite the numerous aforementioned limitations in the literature, it is clear that preterm and full-term infants have the capacity to perceive and respond to pain. ${ }^{[57]}$ It appears that there is the potential for early neonatal pain experience to cause changes in how infants respond to future painful procedures. Some of the strongest evidence for prolonged effects of neonatal pain comes from controlled studies that have tested the preventive effects of analgesics. Administration of analgesic agents (local anesthetics or opioids) prior to noxious procedures both minimized procedural pain and the magnitude of long-term changes in pain behaviors. The precise determinants of these changes, their extent, and their permanence are not known but they appear to involve noxious stimulus-induced peripheral and central sensitization, as well as classical conditioning. Continued investigation is required of these and other factors affecting the experience of early pain on the developing infant.

\section{Acknowledgments}

Dr Taddio is supported by a New Investigator Award from the Canadian Institutes of Health Research. Dr Katz is supported by a Canada Research Chair in Health Psychology at York University, Toronto, Ontario, Canada. The authors have no conflicts of interest that are directly relevant to the content of this review.

\section{References}

1. Grunau RE. Long-term consequences of pain in human neonates. In: Anand KJS. Stevens BJ, McGrath PJ, editors. Pain in neonates: pain research and clinica management. 2nd ed. New York: Elsevier, 2000: 55-76

2. Melzack R, Katz J. Assessment of pain in adult patients. In: McMahon SB Koltzenburg M. editors. Wall \& Melzack's textbook of pain. 5th ed. New York: Churchill-Livingstone, 2005

3. Anand KJ, Craig KD. New perspectives on the definition of pain. Pain 1996; 67: 3

4. McIntosh N. Pain in the newborn, a possible new starting point. Eur J Pediatr 1997 156: $173-7$

5. Morison SJ, Grunau RE, Oberlander TF, et al. Relationships between behaviora and cardiac autonomic reactivity to acute pain in preterm infants. Clin J Pain 2001; $17: 350-8$

6. Grunau RVE, Craig KD. Pain expression in neonates: facial action and cry. Pain 1987: $28: 395-410$

7. Stevens BJ, Johnston CC, Petryshen P, et al. Premature infant pain profile: development and initial validation. Clin I Pain 1996: 12: 13-22

8. Anand P, Birch R. Restoration of sensory function and lack of long-term chronic pain syndromes after brachial plexus in ury in human neonates. Brain 2002 125: $113-22$

9. Birch R, Bonney G, Wynn Parry CB, Birth lesions of the brachial plexus. In: Birch R. Bonney G, Wynn Parry CB, editors. Surgical disorders of the periphera nerves. Edinburgh: Churchill Livingstone, 1998: 209-33

10. McCann ME, Waters P, Goumnerova LC, et al. Self-mutilation in young children following brachial plexus birth injury. Pain 2004; 110: $123-9$

11. Wall PD, Devor M, Inbal R, et al. Autotomy following peripheral nerve lesions: experimental anaesthesia dolorosa. Pain 1979; 7: 103-11

12. Coderre TJ, Grimes RW, Melzack R. Deafferentation and chronic pain in animals: an evaluation of evidence suggesting autetomy is related to pain. Pain 1986; 26 61-84

13. Mailis A. Compulsive targeted self-injurious behaviour in humans with neuropathic pain: a counterpart of animal autotomy? Four case reports and literature review, Pain 1996: 64: 569-78

14. Rossitch Jr E. Oakes WJ, Ovelmen-Levitt J, et al. Self-mutilation following brachial plexus injury sustained at birth. Pain 1992; 50: 209-11 
15. Al-Qattan MM. Self-mutilation in children with obstetric brachial plexus palsy. J Hand Surg 1999; 24: 547-9

16. Grunau RVE, Craig KD, Drummond JE. Neonatal pain behaviour and perinatal events: implications for research observations. Can J Nurs Res 1989; 21 (3): 7 17

17. Gunnar MR. Reactivity of the hypothalamic-pituitary-adrenocortical system to stressors in normal infants and children. Pediatrics 1992; 90 (3): 491-7

18. Gunnar MR. Hertsgaard L, Larson M. Cortisol and behavioral responses to repeated stressors in the human newborn. Dev Psychobiol 1992; 24: 487-505

19. Littman B. Parmelee AH. Medical correlates of infant development. Pediatrics 1978: 61: $470-4$

20. Ramsay DS, Lewis M. The effects of birth condition on infants' cortisol response to stress. Pediatrics 1995; 95 (4): 546-9

21. Taylor A, Fisk NM, Glover V. Mode of delivery and subsequent stress response. Lancet 2000;355: 120

22. Taddio A, Goldbach M, Ipp M, et al. Effect of neonatal circumcision on pain responses during vaccination in boys. Lancet 1995:345: 291-2

23. Taddio A. Katz J, Mersich AL, et al. Effect of neonatal circumcision on pain response during subsequent routine vaccination. Lancet 1997; 349: 599-603

24. Peters JWB, Koot HM, deBoer JB, et al. Major surgery within the first 3 months of life and subsequent biobehavioral pain responses to immunization at later age; a case comparison study. Pediatrics 2003; 111 (1): 129-35

25. Van Dijk M, Bouwmeester NJ, Duivenvoorden HJ, et al. Efficacy of continuous versus intermittent morphine administration after major surgery in 0 to 3-yearold infants; a double-blind randomized controlled trial. Pain 2002; 98: 305-13

26. Keene AR, Cullen DJ. Therapeutic intervention scoring system: update. Crit Care Med 1989:11:1-3

27. Peters JW, Schouw R, Anand KJ, et al. Does neonatal surgery lead to increased pain sensitivity in later childhood? Pain 2005; 114: 444-54

28. van Dijk M, de Boer JB, Koot HM, et al. The reliability and validity of the COMFORT scale as a postoperative instrument in 0 to 3 -year-old infants. Pain 2000: $84: 367-77$

29. Andrews KA, Desai D, Dhillon HK, et al. Abdominal sensitivity in the first year of life: comparison of infants with and without prenatally diagnosed unilateral hydronephrosis. Pain 2002; 100:35-46

30. Grunau R. Early pain in preterm infants: a model of long-term effects. Clin Perinatol 2002; 29: 373-94

31. Johnston CC. Stevens BJ. Experience in a neonatal intensive care unit affects pain response. Pediatrics 1996: 98 (5): 925-30

32. Grunau RE, Oberlander TF, Whitfield MF, et al. Demographic and therapeutic determinants of pain reactivity in very low birth weight neonates at 32 weeks' postconceptional age. Pediatrics 2001; 107 (1): 105-12

33. Fitzgerald M, Millard C, McIntosh N. Cutaneous hypersensitivity following peripheral tissue damage in newborn infants and its reversal with topical anaesthesia. Pain 1989; 39: 31-6

34. Johnston CC, Stevens B, Yang F, et al. Developmental changes in response to heel stick in preterm infants: a prospective cohort study. Dev Med Child Neurol 1996: 38: 438-45

35. Porter FL, Wolf CM, Miller JP. Procedural pain in newborn infants: the influence of intensity and development [online]. Available from URL: http: //www.pediatrics.org/cg/content/full/104/1/e13 [Accessed 2005 Jun 23]

36. Grunau RE, Holsti L. Haley DW, et al. Neonatal procedural pain exposure predicts lower cortisol and behavioral reactivity in preterm infants in the NICU. Pain $2005 ; 113: 292-300$

37. Grunau RE, Weinberg J, Whitfield MF. Neonatal procedural pain and preterm infant cortisol response to novelty at 8 months. Pediatrics 2004: 114: e77-84
38. Whitfield MF, Grunau RE. Behavior, pain perception, and the extremely low birth weight survivor. Clin Perinatol 2000; 27 (2): 363-79

39. Oberlander TF, Grunau RE, Whitfield MF, et al. Biobehavioral pain responses in former extremely low birth weight infants at four months' corrected age [online]. Available from URL: http: //www.pediatrics.org/cgi/content/full/105/ 1/e6 [Accessed 2005 Jun 23]

40. Grunau RE, Oberlander TF, Whitfield MF, et al. Pain reactivity in former extremely low birth weight infants at corrected age 8 months compared with term born controls. Inf Behav Dev 2001; 24: 41-55

41. Buskila D, Neumann L, Zmora E, et al. Pain sensitivity in prematurely born adolescents. Arch Pediatr Adolesc Med 2003; 157: 1079-82

42. Craig KD, Whitfield MF, Grunau RVE, et al. Pain in the preterm neonate behavioural and physiological indices. Pain 1993; 52: 287-99

43. Porter FL, Wolf CM, Miller JP. The effect of handling and immobilization on the response to acute pain in newborn infants. Pediatrics 1998; 102 (6): 1383-9

44. Johnston CC, Stevens BJ, Franck LS, et al. Factors explaining lack of response to heel stick in preterm newborns. J Obstet Gynecol Neonatal Nurs 1999; 28 (6): $587-94$

45. Langland JT, Langland Pl. Letter to the editor. N Engl J Med 1988; 318 (21): 1398 9

46. Brereton RJ. Letter to editor. J Pediatr Surg 1991; 26 (10): 1262

47. McGrath PJ, Craig K. Developmental and psychological factors in children's pain Pediatr Clin North Am 1989; 36 (4): 823-36

48. Owens ME, Todt EH. Pain in infancy: neonatal reaction to a heel lance. Pain 1984: 20: $77-86$

49. Goubet N, Clifton RK, Shah B. Learning about pain in preterm newborns. J Dev Behav Pediatr 2001; 22 (6): 418-24

50. Taddio A, Shah V, Gilbert-MacLeod C, et al. Conditioning and hyperalgesia in newborns exposed to repeated heel lances. JAMA 2002; 288 (7): 857-61

51. Porter FL, Wolf CM, Gold J, et al. Pain and pain management in newborn infants: a survey of physicians and nurses. Pediatrics 1997: 100: 626-32

52. Johnston CC. Collinge JM, Henderson S, et al. A cross-sectional survey of pain and analgesia in Canadian neonatal intensive care units. Clin J Pain 1997: 13 (4): $308-12$

53. Barker DP, Rutter N. Exposure to invasive procedures in neonatal intensive care unit admissions. Arch Dis Child Fetal Neonat Ed 1995: 72: F47-8

54. American Academy of Pediatrics (Committee on Fetus and Newborn; Committee on Drugs; Section on Anesthesiology: and Section on Surgery), Canadian Paediatric Society (The Fetus and Newborn Committee). Prevention and management of stress and pain in the newborn infant. Pediatrics 2000; 105 (2): $454-$ 61

55. International Evidence-Based Group for Neonatal Paim. Consensus statement for the prevention and management of pain in the newborn infant. Arch Pediatr Adolesc Med 2001; 155 (2): 173-80

56. Gressens P, Rogido M, Paindaveine B, et al. The impact of neonatal intensive care practices on the developing brain. J Pediatr 2002; 140: 646-53

57. Anand KJS, Hickey PR. Pain and its effects in the human neonate and fetus. N Engl J Med 1987; 317: 1321-9

Correspondence and offprints: Dr Joel Katz, Acute Pain Research Unit, Department of Anesthesia and Pain Management, Toronto General Hospital, EN 3-440, 200 Elizabeth Street, Toronto, ON, Canada M5G 2C4.

E-mail: jkatz@yorku.ca or anna.taddio@sickkids.on.ca 